\title{
Efficacy of a low-glycaemic index diet in women with polycystic ovary syndrome
}

\author{
S. Barr, S. Reeves, K. Sharp and Y. Jeanes \\ Health Sciences Research Centre, Roehampton University, London SW15 4JD, UK
}

Polycystic ovary syndrome (PCOS) is a common endocrine disorder affecting between 5-10\% of women of reproductive age in the $\mathrm{UK}^{(1)}$. Weight management is the primary strategy for overweight women with PCOS (wPCOS); however, $80 \%$ of wPCOS, including lean women, are insulin resistant ${ }^{(2)}$. Low-glycaemic index (GI) diets are proposed as a useful strategy for the management of insulin resistance in ${ }_{\mathrm{WPCOS}}^{(3)}$. This research aims to determine the efficacy of an isoenergetic low-GI dietary intervention in wPCOS.

Twenty-one wPCOS (mean age 32.1 (SD 6.7)) years completed a 12-week low-GI dietary intervention (weeks 12-24), preceded by a 12-week control period (weeks 0-12). Dietary intake, body composition and measures of insulin sensitivity were determined.

\begin{tabular}{|c|c|c|c|c|c|c|}
\hline & \multicolumn{2}{|c|}{ Week $0\left(\begin{array}{ll}n & 21\end{array}\right)$} & \multicolumn{2}{|c|}{ Week $12(n$ 21) } & \multicolumn{2}{|c|}{ Week $24(n$ 21) } \\
\hline & Mean & SD & Mean & SD & Mean & SD \\
\hline BMI $\left(\mathrm{kg} / \mathrm{m}^{2}\right)$ & 29.0 & 5.3 & 29.1 & 5.0 & 28.5 & 4.5 \\
\hline Dietary GI* & 54.6 & 3.5 & $54.5^{\mathrm{a}}$ & 4.8 & $49.0^{\mathrm{a}}$ & 5.1 \\
\hline Dietary GL $†$ & 10.3 & 3.7 & 10.7 & 3.2 & 9.8 & 5.4 \\
\hline Energy $[\mathrm{kJ} / \mathrm{d}(\mathrm{kcal} / \mathrm{d})]$ & $8493.52(2030)$ & 1615. 024 (386) & $8430.76(2015)$ & $2581.528(617)$ & $8242.48(1970)$ & $2045.976(489)$ \\
\hline $\mathrm{CHO}$ intake ( $\%$ energy) & 43.0 & 8.4 & 46.9 & 8.0 & 46.0 & 8.0 \\
\hline Fat intake ( $\%$ energy) & 38.3 & 6.6 & 35.6 & 5.8 & 34.3 & 5.1 \\
\hline Protein intake ( $\%$ energy) & 16.1 & 3.6 & 15.3 & 3.6 & 16.5 & 3.8 \\
\hline Fasting glucose $(\mathrm{mmol} / \mathrm{l})$ & 5.1 & 0.3 & 5.2 & 0.2 & 5.2 & 0.3 \\
\hline $2 \mathrm{~h}$ glucose $(\mathrm{mmol} / \mathrm{l})$ & 5.5 & 1.1 & 5.2 & 1.0 & 5.6 & 1.5 \\
\hline Fasting insulin $(\mu \mathrm{U} / \mathrm{ml})$ & 13.3 & 5.4 & 14.6 & 6.3 & 12.5 & 5.2 \\
\hline $2 \mathrm{~h}$ insulin $(\mu \mathrm{U} / \mathrm{ml})$ & 42.3 & 21.8 & 40.4 & 25.4 & 41.2 & 26.1 \\
\hline Insulin resistance (HOMA index) & 1.7 & 0.7 & 1.9 & 0.8 & 1.6 & 0.7 \\
\hline Insulin sensitivity (HOMA index) & 69.2 & 34.1 & $61.1^{\mathrm{b}}$ & 24.9 & $72.8^{\mathrm{b}}$ & 32.0 \\
\hline
\end{tabular}

*Low: <55, medium: 55-69, high $\geq 70$; †Low: $<10$, medium: $10-19.9$, high $\geq 20$. ${ }^{(\mathrm{a}, \mathrm{b})} P<0.05$.

At week 24, dietary GI reduced significantly from 54.5 (SD 3.5) at week 12 to 49.0 (SD 5.1) $(P<0.001)$ and insulin sensitivity improved $(P=0.027)$. Trends for improvement to fasting insulin $(P=0.08)$, insulin resistance (HOMA-IR) $(P=0.08)$ and waist circumference $(94.3$ (SD 12.2) $\mathrm{cm}$ to 91.2 (SD 10.7) $\mathrm{cm}(P=0.06)$ ) were found, with no significant changes to weight or macronutrient intake. Percentage carbohydrate and fat intake at baseline were not in line with current recommendations for health; consistent with previous findings ${ }^{(4)}$.

This is the first study to implement an isoenergetic low-GI diet in wPCOS. Initial findings demonstrate improvements to insulin sensitivity independent of weight loss. These results will help to inform dietetic practice and provide essential evidence towards effective dietary management for both lean and overweight women with PCOS.

1. Franks S (1995) Polycystic ovary syndrome. N Engl J Med 333, 853-861.

2. Carmina E \& Lobo RA (2004) Use of fasting blood to assess the prevalence of insulin resistance in women with polycystic ovary syndrome. Fertil Steril 82, 661-665.

3. Marsh K \& Brand-Miller J (2005) The optimal diet for women with polycystic ovary syndrome? Br J Nutr 94, 154-165.

4. Barr S, Hart K, Reeves S et al. (2007) Dietary composition of UK women with polycystic ovary syndrome. Ann Nutr Metab 51, suppl. 1, 345. 